Известия НАН Армении, Физика, т.57, №1, с.116-124 (2022)

УДК 536.2

DOI:10.54503/0002-3035-2022-57.1-116

\title{
ПЕРЕХОД СВЕРХПРОВОДНИК-ИЗОЛЯТОР В УЛЬТРАТОНКИХ НАНОПЛАСТИНАХ $\mathrm{Sb}_{2} \mathrm{Te}_{3}$
}

\author{
А.А. КУЗАНЯН ${ }^{1,3}$, С.Р. АРУТЮНЯН ${ }^{1,2 *}$ \\ ${ }^{1}$ Институт физических исследований НАН Армении, Аштарак, Армения \\ ${ }^{2}$ Российско-Армянский университет, Ереван, Армения \\ ${ }^{3}$ Калифорнийский университет, Лос-Анджелес, Калифорния, США \\ *e-mail: sergeyhar56@gmail.com \\ (Поступила в редакцию 3 ноября 2021 г.)
}

\begin{abstract}
Исследована проводимость монокристаллических нанопластин топологического изолятора $\mathrm{Sb}_{2} \mathrm{Te}_{3}$. В ультратонких нанопластинах $\mathrm{Sb}_{2} \mathrm{Te}_{3}$ при температуре около $4 \mathrm{~K}$ возникает резкое падение сопротивления, которое является проявлением сверхпроводимости. Результаты показывают, что наличие оптимальной степени беспорядка является необходимым условием возникновения сверхпроводимости. В этих нанопластинах наблюдается перестраиваемый магнитным полем переход сверхпроводник-изолятор. Температурная зависимость магнитосопротивления в полях меньше критического $\left(B<B_{C}\right)$ показывает последовательную трансформацию слабой антилокализационной аномалии в сверхпроводящий переход. Получено значение показателя корреляционной длины $v=0.75 \pm 0.05$ с применением теории масштабирования.
\end{abstract}

\section{1. Введение}

При понижении температуры многие металлы переходят из нормального в сверхпроводящее состояние, которое феноменологически характеризуется возможностью протекания бездиссипативного электрического тока и эффектом Мейснера. В результате изменения какого-либо внешнего параметра (например, напряженности магнитного поля), сверхпроводимость может быть уничтожена. В подавляющем большинстве случаев это приводит к возврату сверхпроводящего материала в металлическое состояние. Однако, было обнаружено, что есть электронные системы в которых нарушение сверхпроводимости приводит к переходу в изолятор, а не в нормальный металл. Переходы сверхпроводник-изолятор (SIT) в двумерных материалах реализованные в тонких пленках и интерфейсах, являются примером непрерывного квантового фазового перехода $[1,2]$. Квантовые фазовые переходы происходят при нулевой температуре и включают изменение основного состояния в ответ на изменение внешнего параметра $[1,2]$. Этим параметром может служить параллельное или перпендикулярное магнитное поле, беспорядок (толщина тонких пленок) или плотность заряда. 
После открытия топологических изоляторов, ТИ, стало ясно, что существует сильная корреляция между сверхпроводимостью и природой топологии. Топологические изоляторы - это новый класс квантовых материалов, в которых бесщелевые металлические поверхностные состояния (SS) сосуществуют с объемной запрещенной зоной [3-6]. Поверхностные состояния, состоящие из спиральных безмассовых конусов Дирака с синхронизацией по спину и моменту, защищенные симметрией обращения времени (TR) возникают из-за сильного спин-орбитального (SO) взаимодействия. Замечательным следствием этого является предотвращение обратного рассеяния электронов в поверхностных состояниях, вызываемых дефектами решетки. Еще один важный аспект - возможность наблюдать сверхпроводимость в неупорядоченных 2D-системах. Сверхпроводимость в двумерных материалах позволяет получить и исследовать множество новых и фундаментальных физических явлений $[7,8]$. Эта тема стала еще более актуальной с осознанием того, что высокотемпературные сверхпроводники по своей природе неупорядоченные [9]. К настоящему времени обнаружено ограниченное количество материалов, которые можно отнести к топологическим сверхпроводникам, такие как $\mathrm{Cu}_{\mathrm{x}} \mathrm{Bi}_{2} \mathrm{Se}_{3}[10,11], \mathrm{Bi}_{2} \mathrm{Te}_{3}$ и $\mathrm{Sb}_{2} \mathrm{Te}_{3}$ под высоким давлением [12,13], $\mathrm{In}_{x} \mathrm{Sn}_{1-x} \mathrm{Te}$ [14, 15], $\mathrm{Bi}_{2} \mathrm{Se}_{3}$ [16], $\mathrm{Tl}_{5} \mathrm{Te}_{3}$ [17], $\mathrm{Sr}_{x} \mathrm{Bi}_{2} \mathrm{Se}_{3}$ [18], $\mathrm{SnTe}$ [19], и $\mathrm{Sb}_{2} \mathrm{Te}_{3}$ [20], выращенные под давлением с избыточным количеством Те.

Целью настоящей работы являлось выявление корреляции между толщиной нанопластин $\mathrm{Sb}_{2} \mathrm{Te}_{3}$ и их проводимостью. Уменьшение толщины выращенных нанопластин привело к возникновению в них сверхпроводимости. Сверхпроводимость, полученная в наших $\mathrm{Sb}_{2} \mathrm{Te}_{3}$ образцах, полученная лишь изменением их толщины открывает новые пути для понимания взаимосвязи между топологическими поверхностными состояниями и сверхпроводимостью.

\section{2. Экспериментальные результаты и их обсуждение}

Исследованное в данной работе монокристаллическое соединение $\mathrm{Sb}_{2} \mathrm{Te}_{3}$ не относится к сверхпроводникам и является надежным топологическим изолятором. Соединение имеет кристаллическую структуру с пространственной группой симметрии $R \overline{3 m}$. Монокристалл $\mathrm{Sb}_{2} \mathrm{Te}_{3}$ это полупроводник $p$-типа (вакансии $\mathrm{Sb}$ и дефекты антипозиции $\mathrm{Sb}_{\mathrm{Te}}$ ответственны за генерацию дырок), а точка Дирака в энергетическом спектре расположена выше энергии Ферми $E_{F}$.

Образование нанопластин происходило при остывании пара исходного материала (в данном случае $\mathrm{Sb}_{2} \mathrm{Te}_{3}$ ) в зоне конденсации на подложке $\mathrm{SiO}_{2} / \mathrm{Si}$. Испарение и конденсация происходили в вакууме $\sim 10^{-6}$ рт. ст. Атомное отношение $\mathrm{Sb} /$ Те соответствует $(40 \pm 1) /(60 \pm 1)$.

Выращенные нанопластины различной толщины показаны на рис.1. Измерение электрического сопротивления нанопластины в зависимости от температуры, $R(T)$, проводилось четырехконтактным методом на установке по измерению физических свойств (PPMS) в магнитных полях до 9 Т и температуpax от 2 К до 300 К. Геометрия расположения электрических контактов показана 

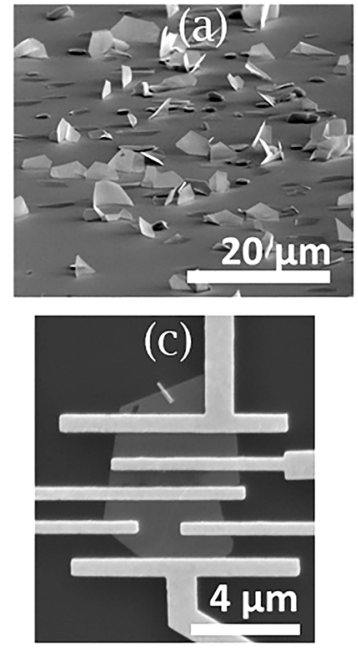

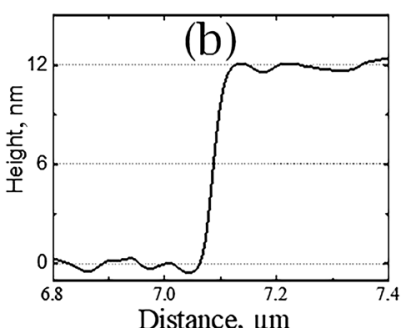

Distance, $\mu \mathrm{m}$

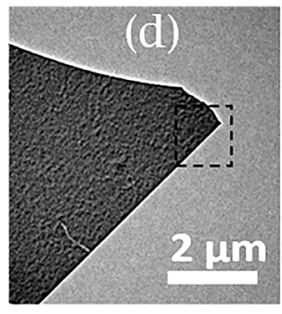

Рис.1. ( а) выращенные на подложке $\mathrm{SiO}_{2} / \mathrm{Si}$ нанопластины различной толщины, (b) профиль толщины нанопластины NF12 полученный атомным силовым микроскопом (AFM), (c) нанопластина NF12 с нанесенными электродами (d) часть нанопластины подтверждающая наличие макроскопических дефектов. Цифры при NF показывают толщину нанопластины в нанометрах.

на рис.1с. Методы энергодисперсионной рентгеновской спектроскопии EDX и просвечивающей электронной микроскопии ТEM, были применены для контроля качества полученных нанопластин. Выращенные нанопластины характеризуются как монокристаллы с однородным пространственным распределением $\mathrm{Sb}$ и Те. $R(T)$ измерения демонстрируют возникновение сверхпроводимости в ультратонких монокристаллических нанопластинах $\mathrm{Sb}_{2} \mathrm{Te}_{3}$. Двумерная сверхпроводимость, обнаруженная в этих ультратонких образцах, приводит к перестраиваемому магнитным полем переходу сверхпроводник-изолятор (SIT). Кривые нормированного сопротивления $R(T) / R 300$ для некоторых нанопластин разной толщины, где $R 300$ - сопротивление при 300 К, были приведены в предыдущей нашей работе, [22]. Уменьшение наклона кривых $R(T) / R 300$ с толщиной является следствием увеличения беспорядка (уменьшение толщины) в образцах. Сопротивление нанопластин тоньше 27 нм резко падает около 4 К, которое мы связываем с началом сверхпроводящего перехода. Величина относительного падения сопротивления при сверхпроводящем переходе зависит от степени неупорядоченности (толщины) нанопластин. Однако степень беспорядка не коррелирует однозначно с толщиной и удельным сопротивлением нанопластин (по крайней мере, в пределах поправки на несколько нанометров). Это может быть результатом непреднамеренных повреждений (трещины, дыры, частично оторванная поверхность, см. рис.1d), накопленных во время подготовки образцов для проведения измерений. Таким образом, мы рассматриваем нормированное сопротивление как наиболее надежный параметр для сравнения различных нанопластин. Для нанопластин со сверхпроводящим переходом кривые $R(T) / R 300$ в 
области сильного электрон-фононного взаимодействия (50-300 К) ведут себя как степенная функция $R \sim T^{\alpha}$, где параметр $\alpha$ изменяется (в зависимости от толщины) в пределах 0.4-1.25. Для образцов с наибольшим содержанием сверхпроводящей фазы, имеющих наибольшее относительное изменение сопротивления $\left(R_{4 \mathrm{~K}}-R_{2 \mathrm{~K}}\right) / R_{2 \mathrm{~K}}$ при сверхпроводящем переходе (рис.2), значение параметра $\alpha$ изменяется в пределах $0.65 \pm 0.03$, что предполагает наличие оптимальной степени беспорядка.

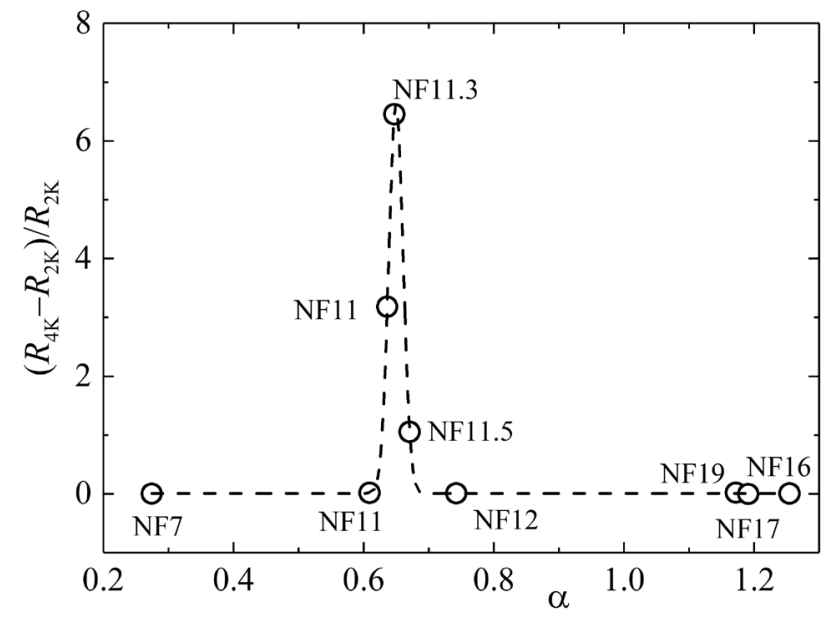

Рис.2. Относительное изменение сопротивления $\left(R_{4 \mathrm{~K}}-R_{2 \mathrm{~K}}\right) / R_{2 \mathrm{~K}}$ при сверхпроводящем переходе для разных по толщине нанопластин в зависимости от параметра $\alpha$.

В сильных магнитных полях носители заряда в ультратонких нанопластинах локализуются из-за подавления сверхпроводимости и нарушения симметрии обращения времени (TR). Эволюция продольного сопротивления $R_{x x}(B)$ (типичного для всех нанопластин, испытывающих сверхпроводящий переход) с температурой в поперечном магнитном поле для образца NF7 продемонстрирована на рис.3.

Кривые $R_{x x}(B)$ пересекаются в определенной области магнитного поля $B_{\mathrm{C}}$ (критическое поле) и в конечном итоге выстраиваются в обратном порядке при полях $B>B_{\mathrm{C}}$, то есть магнитосопротивление в данном магнитном поле уменьшается с увеличением температуры. Это проявление эффекта слабой локализации (WL) и индуцированного магнитным полем перехода сверхпроводник-изолятор (SIT) для двумерного случая, наблюдаемое в тонких пленках. Переход представляет собой квантовый фазовый переход, в котором преобладают квантовые флуктуации и контролируются ими [1,2]. Настройка SIT достигается приложением поперечного магнитного поля или изменением толщины пленки. Магнитное поле переводит сверхпроводящее состояние неупорядоченной пленки в слабых полях через металлическое состояние при критическом поле $B_{\mathrm{C}}$ в изолирующее состо-

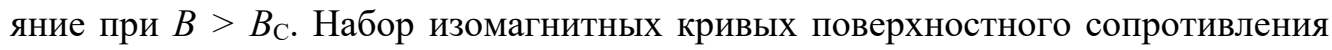
образца NF7 в различных поперечных магнитных полях, показан на рис.4. 


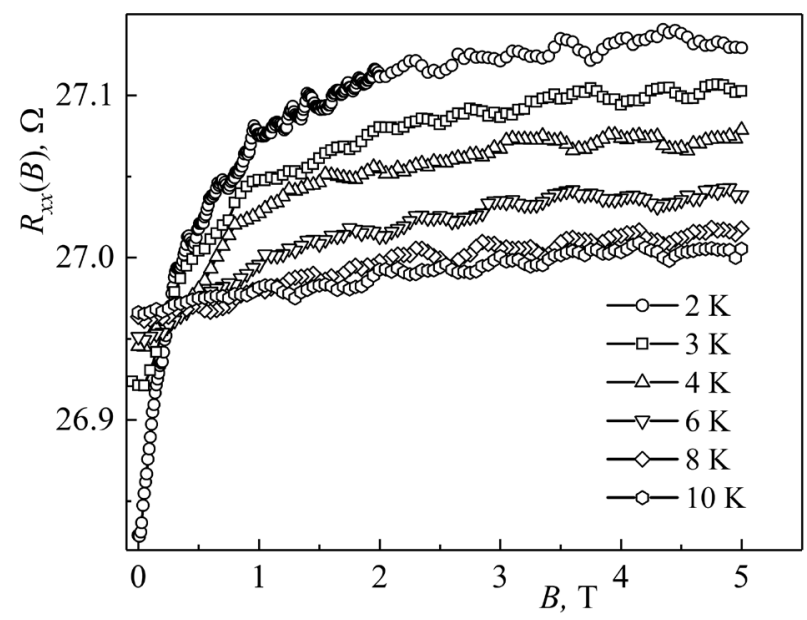

Рис.3. Зависимость продольного сопротивления $R_{x x}(B)$ (типичного для всех нанопластин, испытывающих сверхпроводящий переход) от температуры в поперечном магнитном поле образца NF7.

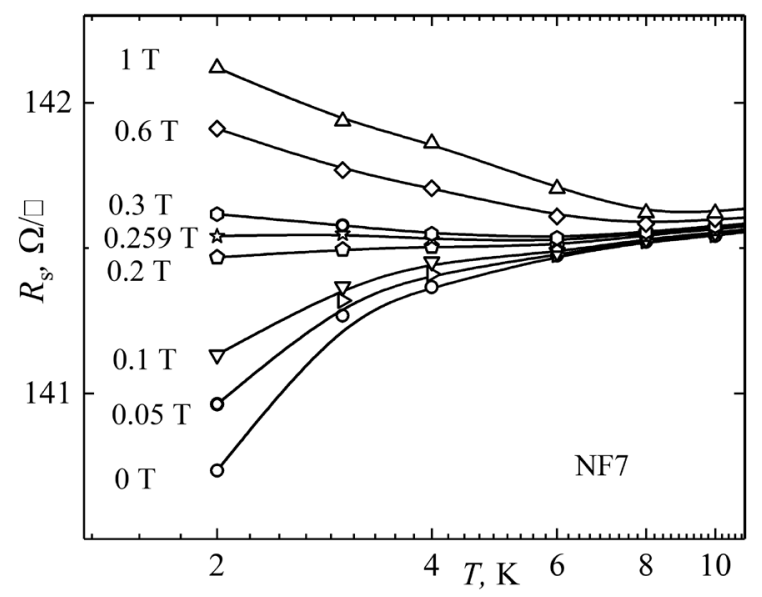

Рис.4. Изомагнитные кривые поверхностного сопротивления $R_{\mathrm{S}}$, образца NF7 при различных поперечных магнитных полях.

Эти зависимости можно разделить на две группы по знаку производной $d R / d T$. Положительный (отрицательный) знак соответствует сверхпроводящему (изолирующему) поведению. Граничная изомагнитная кривая $R_{\mathrm{C}}(T)$ между фазами сверхпроводника и диэлектрика соответствует металлическому состоянию при $T=0 \mathrm{~K}$ и показывает величину критического поля $B_{\mathrm{C}}$, которая для данного образца составляет $B_{\mathrm{C}}=0.259$ Тл.

График зависимости поверхностного сопротивления $R_{\mathrm{S}}$ от масштабной переменной $\left|B-B_{\mathrm{C}}\right| / T^{1 / z v}$, где $v$ - показатель корреляционной длины, а $z$ - показатель динамического масштабирования, приведен на рис.5.

На графике четко видны две ветви сверхпроводящего и диэлектрического состояния образца. Произведение критических показателей меняется в диапазоне 


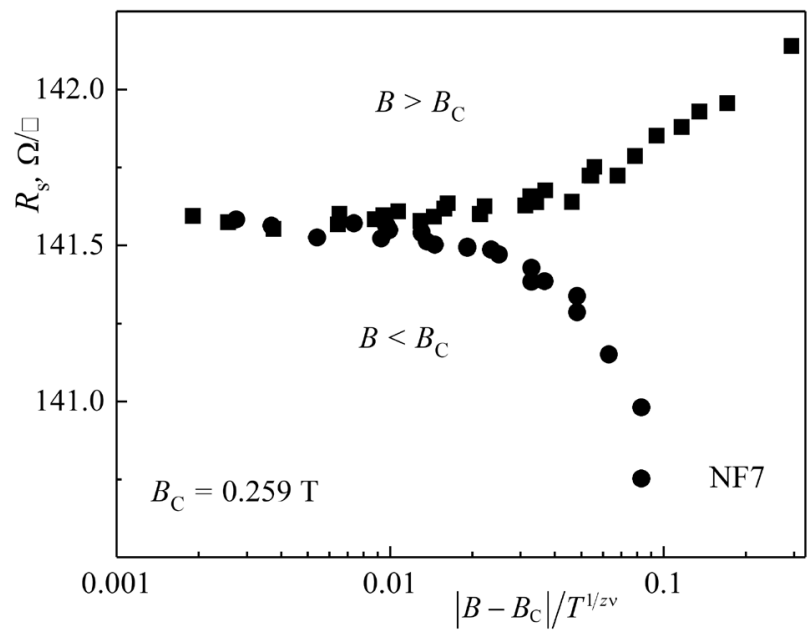

Рис.5. Зависимость поверхностного сопротивления $R_{\mathrm{S}}$ от масштабной переменной $\left|B-B_{\mathrm{C}}\right| / T^{1 / 2 v}$ образца NF7.

$z v=0.75 \pm 0.05$ для разных образцов и выявляется путем наилучшего схождения (колапса) между данными измерений. При конечной температуре размер квантовых флуктуаций ограничен длиной сбоя фазы $L_{\varphi} \propto T^{-1 / z}$, где $z$ ожидается равной 1 для SIT. Полагая $z=1$, получаем $v=0.75 \pm 0.05$, что близко к значениям, полученным для аморфных пленок Ві [21]. Значение показателя не согласуется с теорией масштабирования, которая предсказывает $v>1$. Однако, предыдущие исследования SIT показали, что, когда произведение показателей определяется варьированием магнитного поля, а не толщины образца как параметра настройки, то $z v \approx 0.7 \pm 0.2$. Значение критического сопротивления $R_{\mathrm{C}}$ нанопластин варьируется в диапазоне от 100 Ом/ до 200 Ом/ в вависимости от образца и отклоняется от универсального значения $R_{Q}=h / 4 e^{2} \approx 6.5$ кОм/ロ, предсказываемого теорией масштабирования, и отражает наличие дополнительного проводящего канала.

Полученные кривые $R(T)$ (рис.2) показывают не только наличие некоторого оптимального значения беспорядка (локализации объемных носителей), но и некоторую необходимую для возникновения сверхпроводящего перехода степень беспорядка. Другими словами, при прочих равных условиях существует толщина (беспорядок), при котором объемные состояния частично локализуются и куперовские пары начинают образовываться. Эта ситуация означает повышенный вклад поверхностных состояний в тонких образцах по сравнению с более толстыми образцами. В самых тонких образцах наблюдается ослабление сверхпроводимости [22]. Причиной такого поведения может быть избыточный беспорядок и ослабление влияния поверхностных состояний, вызванное глубокой гибридизацией между верхним и нижним поверхностными состояниями. Когда длина проникновения $\xi=h v_{f} / 2 \pi M\left(M-\right.$ объемная запрещенная зона, а $v_{\mathrm{f}}-$ скорость Ферми) становится сопоставимой с толщиной пленки, в точке Г зоны Брилюена 
открывается щель $\Delta$, и дисперсия поверхностных состояний изменяется на дираковские гиперболы, что приводит к переходу WL. Обращенная во времени петля рассеяния электронов эквивалентна перемещению электрона по поверхности Ферми за один цикл. Как результат, электрон набирает фазу Берри [23]. Фаза Берри $\phi$, приобретаемая электронами при движении по замкнутым траекториям, равна $\pi\left(1-\Delta /\left(2 E_{\mathrm{f}}\right)\right.$, где $E_{\mathrm{f}}$ энергия Ферми, $\Delta-$ щель в энергетическом спектре поверхностных состояний. В случае $\Delta=0$ (невозмущенные поверхностные состояния) фаза Берри $\phi=\pi$, что приводит к слабой антилокализации. При гибридизации $(\Delta \neq 0)$ следует ожидать кроссовера со слабой локализацией. Можно предположить, что эффект WL в наших нанопластинах является результатом дискретизации непрерывного объемного состояния или гибридизации поверхностных состояний, что, вероятно, является следствием комбинированного воздействия этих двух процессов. Таким образом, можно предположить, что совместное влияние локализованных объемных состояний и поверхностных состояний является необходимым условием возникновения 2D сверхпроводимости в топологическом изоляторе $\mathrm{Sb}_{2} \mathrm{Te}_{3}$. Предполагая сравнимые скорости Ферми в $\mathrm{Sb}_{2} \mathrm{Te}_{3}$ и $\mathrm{Bi}_{2} \mathrm{Se}_{3}$, и учитывая, что $\mathrm{Sb}_{2} \mathrm{Te}_{3}$ имеет меньшую объемную запрещенную зону, чем $\mathrm{Bi}_{2} \mathrm{Se}_{3}$, то гибридизация верхнего и нижнего поверхностных состояний будет достигнута у $\mathrm{Sb}_{2} \mathrm{Te}_{3}$ в более толстых образцах, чем в случае с $\mathrm{Bi}_{2} \mathrm{Se}_{3}$. Предел толщины пленки $\mathrm{Bi}_{2} \mathrm{Se}_{3}$, в которой сохраняются поверхностные состояния, составляет всего 9.0 нм. Это означает, что вблизи толщин 10 нм можно ожидать гибридизации и в образцах $\mathrm{Sb}_{2} \mathrm{Te}_{3}$.

Наблюдаемое начало сверхпроводимости происходит при низких температурах 4 К, что предполагает наличие небольшой сверхпроводящей щели, которая может быть сопоставима только с гибридизационной щелью поверхностных состояний, но не с объемной щелью ( $>0.1$ эВ) даже в дискретизированном состоянии. Несмотря на очевидные отклонения параметров $R_{C}$ и $v$ от предсказываемых теорией SIT, основные характеристики квантового SIT, т.е. критическая точка и масштабированное поведение, сохраняются для нанопластин $\mathrm{Sb}_{2} \mathrm{Te}_{3}$, что свидетельствует о существовании сверхпроводимости в образцах. Необходимо подчеркнуть, что наши образцы представляют собой высококачественные монокристаллы, выращенные одновременно, с одинаковым составом. Единственное отличие - это размер, где важнее всего толщина. Поэтому наблюдаемые эффекты мы связываем с изменением их толщины. Существование кроссовера WL - WAL и превращение WAL в SC-переход в $\mathrm{Sb}_{2} \mathrm{Te}_{3}$ уникально и открывает новые пути для понимания взаимосвязи между топологическими поверхностными состояниями и сверхпроводимостью. Температурно-зависимые изомагнитные кривые, отчетливо демонстрируют постепенное превращение WAL эффекта в сверхпроводящий переход. 


\section{3. Заключение}

Транспортные свойства нанопластин топологического изолятора $\mathrm{Sb}_{2} \mathrm{Te}_{3}$ разной толщины, выращенных методом парофазного осаждения показали, что в ультратонких образцах возникает двумерная сверхпроводимость и происходит перестраиваемый магнитным полем переход сверхпроводник-изолятор. Обнаружена последовательная трансформация сверхпроводящего перехода в чистый эффект WAL с повышением температуры. Предполагается, что взаимное влияние локализации объемных носителей и поверхностных состояний является необходимым условием возникновения 2D сверхпроводимости в топологическом изоляторе $\mathrm{Sb}_{2} \mathrm{Te}_{3}$.

Авторы благодарны профессору Yang Yuan Chen за предоставленную возможность провести измерения в Институте физики (Тайбэй, Тайвань).

\section{ЛИТЕРАТУРА}

1. A.M. Goldman. Inter. J. of Mod. Phys. B, 24, 4081 (2010).

2. V.F. Gantmakher, V.T. Dolgopolov. Physics-Uspekhi, 33, 1 (2010).

3. M.P.A. Fisher. Phys. Rev. Lett., 67, 923 (1990).

4. M. Sato, Y. Ando. Rep. Prog. Phys., 80, 076501 (2017).

5. H. Zhang, Ch-X. Liu, X-L. Qi, Xi. Dai, Zh. Fang, Sh-Ch. Zhang. Nature Phys., 5, 438 (2009).

6. J. Zhang, C.-Z. Chang, Z. Zhang, J. Wen, X. Feng, K. Li, M. Liu, K. He, L. Wang, X. Chen, Q.-K. Xue, X. Ma, Y. Wang. Nature Commun., 2, 574 (2011).

7. M.Z. Hasan, C.L. Kane. Rev. Modern Phys., 82, 3045 (2011).

8. X-L. Qi, Sh-Ch. Zhang. Rev. Mod. Phys., 83, 1057 (2011).

9. L. Fu, C.L. Kane. Phys. Rev. Lett., 100, 096407 (2008).

10. S., Sasaki, M. Kriener, K. Segawa, et al., Phys. Rev. Lett., 107, 217001 (2011).

11. Y.S. Hor, A.J. Williams, J.G. Checkelsky, et al., Phys. Rev. Lett, 104, 057001 (2010).

12. K. Matsubayashi, T. Terai, J.S. Zhou, Y. Uwatoko, Phys. Rev. B, 90, 125126 (2014).

13. J. Zhu, J. Zhang, P. Kong, et al., Sci. Rep., 3, 2016. (2013).

14. I.F. Herbut, and L. Janssen, Phys. Rev. Lett., 113, 106401 (2014).

15. M.-X. Wang, C. Liu, J.-P. Xu, et al., Science, 336(6077), 52 (2012).

16. R.D. Zhong, J.A. Schneeloch, X.Y. Shi, et al., Phys. Rev. B, 88, 020505(R) (2013).

17. K.E. Arpino, D.C. Wallace, Y.F. Nie, T. Birol, P.D.C. King, S. Chatterjee, M. Uchida, S.M. Koohpayeh, J.J. Wen, K. Page, C.J. Fennie, K.M. Shen, T.M. McQueen. Phys. Rev. Lett., 112, 017002 (2014).

18. Z. Liu, X. Yao, J. Shao, M. Zuo, L. Pi, S. Tan, C. Zhang, Y. Zhang. J. Am. Chem. Soc., 137, 10512 (2015).

19. S. Sasaki, K. Segawa, Y. Ando. Phys. Rev. B: Condens., 90, 220504 (2014).

20. L. Zhao, H. Deng, I. Korzhovska, M. Begliarbekov, Zh. Chen, E. Andrade, E. Rosenthal, A. Pasupathy, V. Oganesyan, L. Krusin-Elbaum. Nat. Commun., 6, 8279 (2015). 
21. A.M. Goldman, N. Marković. Phys. Today, 51(11), 39 (1998).

22. A.A. Kuzanyan, S.R. Harutyunyan. J. Contemp. Phys., 56, 359 (2021).

23. H-Z. Lu, S-Q. Shen. arXiv:1409.1299v1 [cond-mat.mes-hall] 2014.

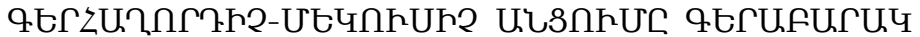

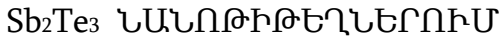

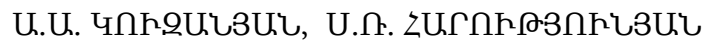

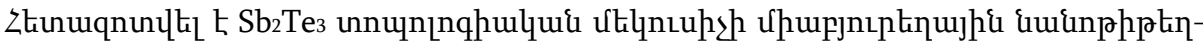

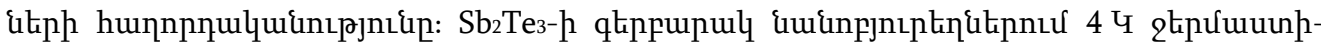

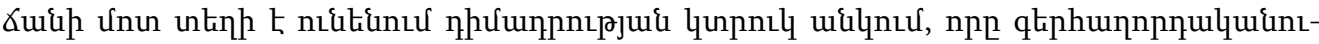

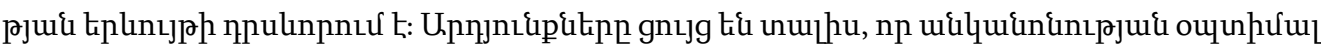

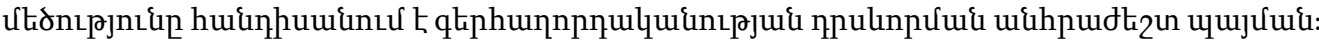

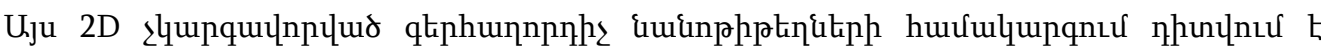

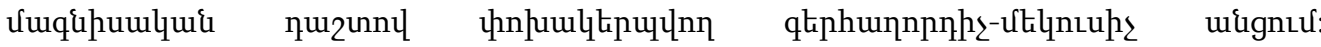

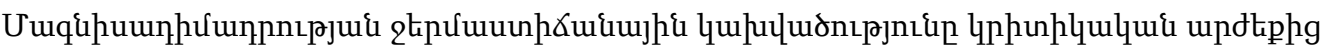

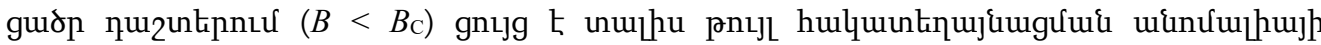

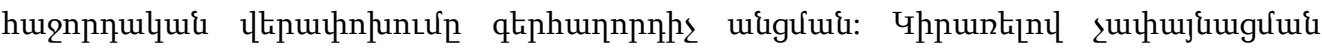

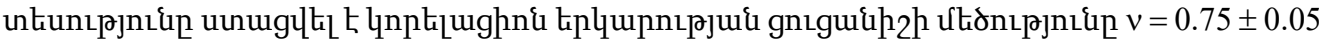

\section{SUPERCONDUCTOR-INSULATOR TRANSITION IN ULTRATHIN $\mathrm{Sb}_{2} \mathrm{Te}_{3}$ NANOPLATES}

\section{A.A. KUZANYAN, S.R. HARUTYUNYAN}

The conductivity of single-crystal nanoplates of the $\mathrm{Sb}_{2} \mathrm{Te}_{3}$ topological insulator is investigated. In ultrathin $\mathrm{Sb}_{2} \mathrm{Te}_{3}$ nanoplates at a temperature of about $4 \mathrm{~K}$, a sharp drop in resistance occurs, which is a manifestation of superconductivity. The results show that the presence of a certain optimal degree of disorder is a necessary condition for the onset of superconductivity. A magnetic-field tunable superconductor - insulator transition in a disordered 2D superconducting system is observed in these nanoplates. The temperature dependence of the magnetoresistance in the fields less than the critical one $\left(B<B_{\mathrm{C}}\right)$ shows a consistent transformation of the weak antilocalization anomaly into the superconducting transition. The value of the correlation length exponent $v=0.75 \pm 0.05$ was found using the scaling theory. 